\title{
A Scoring System for the Early Detection of Oral Submucous Fibrosis Based on a Self-administered Questionnaire
}

\author{
Chung-Jung Chiu, DDS, PhD; Wen-Chung Lee, MD, PhD; Chun-Pin Chiang, DDS, PhD; \\ Liang-Jiunn Hahn, DDS, PhD; Ying-Shiung Kuo, DDS, PhD; Chien-Jen Chen, ScD
}

\begin{abstract}
Objectives: The aims of the present study were to evaluate the frequent clinical complaints of oral submucous fibrosis (OSF) and to develop a scoring system for early detection of the disease by a self-administered questionnaire. Methods: $A$ total of 296 subjects were recruited, including 123 OSF patients without oral cancer and 173 betel quid chewers without OSF or oral cancer. A self-administered questionnaire was used to collect the symptom profile from study subjects. Their maximal mouth opening (MMO) between upper and lower incisor edges was measured and recorded by well-trained nurses. A binary logistic regression model examining the likelihood of OSF based on the eight symptoms of interest was used to develop the scoring system. Results: Among 79 OSF subjects with an MMO $<35 \mathrm{~mm}$, the most frequent complaint was trismus $(87.3 \%)$, followed by burning sensation (76.0\%) and xerostomia (72.2\%). Among 44 OSF subjects with an $M M O \geq 35 \mathrm{~mm}$, burning sensation (68.2\%) was the most frequent complaint, followed by trismus (54.5\%) and xerostomia (54.5\%). Six frequent complaints including trismus, burning sensation, xerostomia, sore throat, numbness, and oral ulceration were utilized to develop a scoring system for the early detection of OSF. The scoring system had an area under the receiver operating characteristic curve of 0.90 . Conclusion: This study suggests a screening questionnaire of frequent complaints for the early detection of OSF. [J Public Health Dent 2002;62(1):28-31]
\end{abstract}

Key Words: oral submucous fibrosis, early detection.

Oral submucous fibrosis (OSF) is a betel quid chewing-specific precancerous condition $(1,2)$. The onset of the disease is often insidious and its clinical symptoms are variable. It may eventually lead to severe mouth opening limitation, trismus (3). Several remedies have been tried to improve the mouth opening of OSF patients with trismus, but none of them was satisfactory (4). Because of the malignant tendency and possible trismus complication, regular follow-up of OSF patients is recommended.

This disease affects approximately 0.5 percent ( 5 million people) of the population in the Indian subcontinent and is now a public health issue in many parts of the world, including the United Kingdom, South Africa (2), and many Southeast Asian countries. In
Taiwan, betel quid chewing was prevalent among the working classes in the past years. Recently, the betel quid chewing population has been growing. The socioeconomic level of chewers is also going up and there are more young betel quid chewers (BQCs). In Taiwan, the impact of OSF is becoming a pressing public health issue. We conducted this study to evaluate frequent clinical complaints of OSF and to develop a scoring system for the early detection of OSF by a self-administered questionnaire. The performance of the scoring system was also evaluated.
Methods
Study Subjects. The consecutive OSF patients were recruited from the Department of Dentistry of National

Taiwan University Hospital from January 27 to December 31, 1999. Every male patient was asked to fill out a one-page questionnaire before clinical examination. The questionnaire included basic demographic characteristics, eight frequent symptoms of OSF, as well as betel quid chewing history. The eight symptoms included trismus (mouth opening limitation), burning sensation while eating spicy food, xerostomia, maltaste (defective gustatory sensation), sore throat or pain in ear region, oral numbness, oral ulceration, and dysphagia. The English version of these eight questions is shown in Table 1.

The maximal mouth opening (MMO) between upper and lower incisor edges of each study subject was measured and recorded by welltrained nurses. Every patient was examined and diagnosed by oral pathologists. The oral pathologists were blinded to the results of the questionnaire. Palpable or strip fibrous bands in a chewer involving one or more oral structures, including buccal mucosa, labial mucosa, fauces, soft palate including the uvula, floor of the mouth, and the tongue were regarded as OSF (5). Scars and oral frenulae were not considered as fibrous bands. Oral symptoms were not regarded as diagnostic for OSF in the absence of fibrous bands. A total of 123 consecutive OSF patients without oral cancer were recruited. To prevent a keloid-like response after biopsy surgery, further histopathologic confirmation was carried out on 31 ( 25.2 percent) patients with suspected malignant transformations. All OSF subjects were BQCs.

One hundred and seventy-three BQCs who had never been diagnosed

Send correspondence and reprint requests to Dr. Chen, Graduate Institute of Epidemiology, College of Public Health, National Taiwan University, 1 Jen-Ai Road Section 1, Taipei 10018, Taiwan. Email: cjchen@ha.mc.ntu.edu.tw. Drs. Chiu and Lee are both affiliated with the Graduate Institute of Epidemiology, College of Public Health, and Drs. Chiu, Chian, Hahn, and Kuo are with the School of Dentistry, College of Medicine, all at National Taiwan University, Taipei. This study was supported by Department of Health grant \#NHRI-GT-EX89P802P and a National Science Council grant \#NSC 89-2314-B002-369. Manuscript received: 2/12/01; returned to authors for revision: 4/18/01; accepted for publication: 7/6/01. 
with OSF or oral cancer were also recruited from five townships including Makung, Chutung, Potzu, Kaoshu, and Sanchi. These townships were selected for the cooperation of local health personnel and residents. They also represented both urban and rural areas in Taiwan. The same questionnaire was used to collect the information from these community subjects.

\section{Statistical Analysis}

Development of a Scoring System for Early Detection. We used a binary logistic regression model to examine the likelihood of being affected with OSF based on the eight symptoms of interest. To develop the model, we utilized the SAS/LOGISTIC program with the data set of 44 OSF subjects having an $\mathrm{MMO} \geq 35 \mathrm{~mm}$ (5-7) and 173 community BQCs. The initial model was developed from the eight symptoms. None of the unaffected BQCs complained of maltaste or dysphagia. These two symptoms of interest, maltaste and dysphagia, were dropped from the model to avoid the problem of being unable to derive fixed estimates. The remained six symptoms were included in the final model. The intercept estimated from the model was subtracted by log (prior odds of OSF), that is, $\log (44 / 173)=-1.369(8)$.

TABLE 1

Questionnaire for Early Detection of Oral Submucous Fibrosis

\begin{tabular}{lll}
\hline 1. Do you often feel stiff cheeks and difficulty in opening & Yes & No \\
mouth? & & \\
2. Do you often feel burning sensation in mouth while eating & Yes & No \\
hot or spicy food? & & \\
3. Do you frequently feel dryness in mouth? & Yes & No \\
4. Do you frequently feel change of taste? & Yes & No \\
5. Do you frequently feel pain in ear/throat region? & Yes & No \\
6. Do you frequently feel numbness in mouth? & Yes & No \\
7. Do you frequently have vesicles and/or ulceration in mouth? & Yes & No \\
8. Do you frequently feel difficulty in swallowing? & & \\
\hline
\end{tabular}

FIGURE 1

Receiver Operating Characteristic (ROC) Curve for Initial Model [Area Under ROC Curve $(A U C)=0.903$ ]

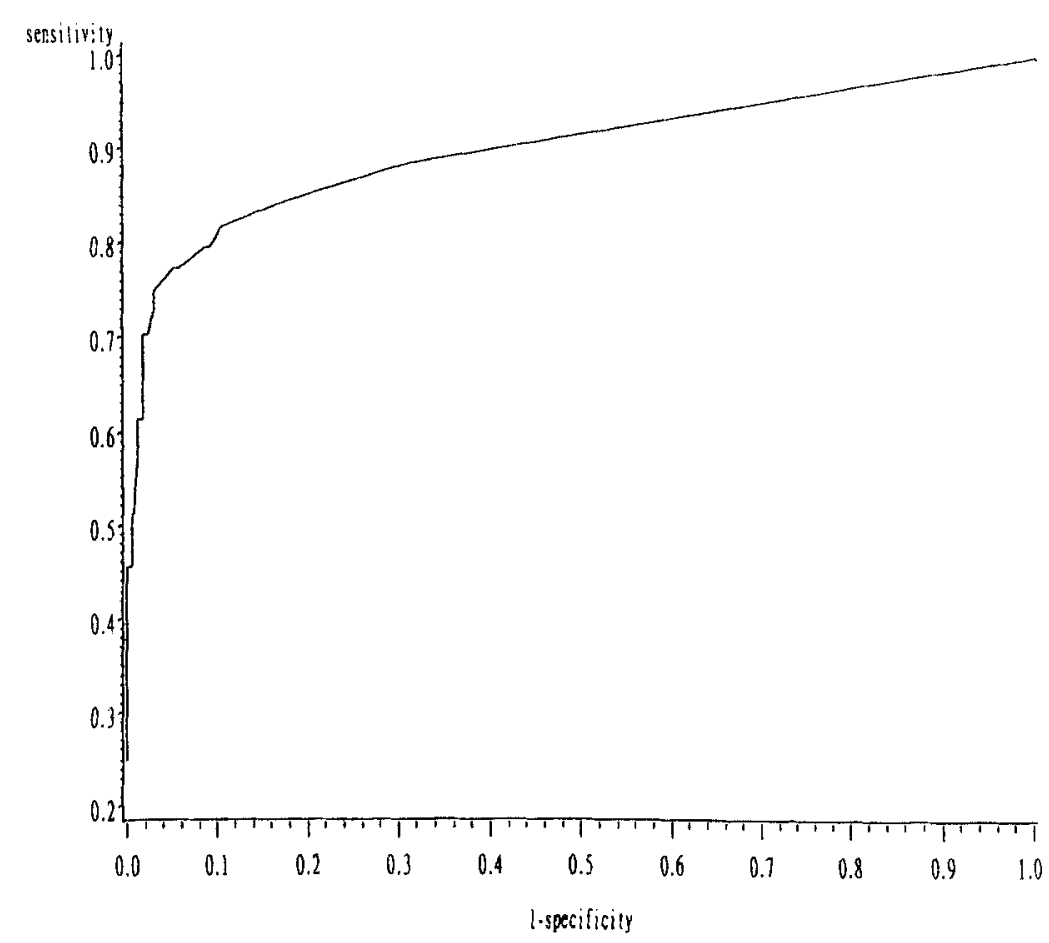

All the estimated parameters in th model were then exponentiated an rounded. A composite score for eacl study subject was obtained by mult plying the scores according to whethe or not the subject had a particula symptom. This composite score repre sented the model-based likelihood $r a$ tios (LRs) of being an OSF case for the particular subject with the specifi combination of symptoms (8). Accorc ing to this scoring system, a study sut ject was classified as a case or noncas if his composite score (the mode based LR) was greater or less than th cutoff point selected to achieve a df sired level of sensitivity and/or spec ficity.

Evaluation of the Scoring Systen To evaluate the screening value of th scoring system, we drew the receive operating characteristic (ROC) curve of the logistic regression model ( 9 The trapezoid rule was utilized to ca culate the areas under ROC curve (AUC) (9). The sensitivities and spec ficities of the scoring system at variot composite score cutoff points were ca culated. Linear intrapolation was af plied to estimate the sensitivity of th scoring system at a particular level specificity if necessary. However, $n$ used the sensitivity to indicate th probability of correct classification , OSF patients, and the specificity to is dicate the probability of correct class fication of unaffected BQCs. To gene ate the ROC curves (Figure 1) of th logistic regression model includin eight symptoms, an OSF subject wh had the symptoms of maltaste and /c dysphagia was classified as an OS case when the probability level wi less than 1.0.

\section{Results}

Demographic Characteristics. TH age range for 123 OSF subjects and 17 unaffected BQCs were 17-66 yea: and 39-72 years, respectively. With $r$ gard to the ethnicity, $100(81.3 \%)$ Os subjects and $140(80.9 \%)$ unaffecte BQCs were Fukien Taiwanese. Wi regard to the education level, all of or study subjects were literate or had $r$ less than an elementary school educ tion.

Symptom Profile of OSF Patien1 The number and percentage of clinic symptoms among the study subjec are listed in Table 2. All of the eig. symptoms were more prevale among OSF cases than among una 
fected BQCs. Among OSF patients with $\mathrm{MMO} \geq 35 \mathrm{~mm}$, burning sensation $(68.2 \%)$, trismus $(54.5 \%)$, xerostomia $(54.5 \%)$, and ulcer $(47.7 \%)$ were the most prevalent symptoms. Among OSF patients with MMO $<35 \mathrm{~mm}$, trismus $(87.3 \%)$ was the most prevalent symptom that differed significantly $(P<.001)$ from the prevalence among OSF patients with $M M O \geq 35 \mathrm{~mm}$ $(54.5 \%)$. The proportions of xerostomia and dysphagia were also significantly $(P<.05)$ different between these two groups of OSF patients.

Screening of OSF with the Scoring System. The scoring system derived from the logistic regression model using six symptoms is shown in Table 3. For a $B Q C$ having complaints of burning sensation and oral ulceration, his composite score was $0.17 \times 4.84 \times$ $2.57=2.11$, which was also the LR of being affected with OSF. The background score $(0.17)$ was the baseline LR of being affected with OSF. Complaint of any symptom increased the composite score. Trismus and burning sensation had more contribution on composite score than the other four symptoms. Xerostomia, sore throat, numbness, and ulceration of oral cavity had similar load on composite score.

The sensitivity and specificity of the scoring system at various cutoff points of the composite score are shown in Table 4 . When the cutoff point was set as 1.0 , the sensitivity and specificity of detecting early-stage OSF were 82.0 percent and 85.8 percent, respectively.

The ROC curve for the model including eight symptoms is shown in Figure 1. The AUC of the ROC curve was 0.903 . The ROC curve for the model including six symptoms was similar to that of the model with eight symptoms showing an AUC of 0.895 (Figure 2).

\section{Discussion}

The eight symptoms investigated in this study have been documented to be prevalent among OSF patients $(3,5)$. According to our data, proportions of these symptoms are significantly $(P<.001)$ higher among OSF patients than among unaffected BQCs. This result implied that the eight symptoms are useful to differentiate OSF patients from unaffected BQCs. Moreover, trismus was the most important symptom for an early-stage OSF patient (MMO $\geq 35 \mathrm{~mm}$ ) to be differentiated from ad-

TABLE 2

Distributions of Clinical Symptoms of 123 Patients Affected with Oral Submucous Fibrosis (OSF) and 173 OSF-free Betel Quid Chewers (BQC)

\begin{tabular}{|c|c|c|c|}
\hline \multirow[b]{2}{*}{ Symptoms } & \multicolumn{2}{|c|}{ OSF $(n=123)$} & \multirow{2}{*}{$\begin{array}{c}\begin{array}{c}\mathrm{BQC} \\
(n=173)\end{array} \\
\text { No. }(\%)\end{array}$} \\
\hline & $\begin{array}{c}\mathrm{MMO}<35 \mathrm{~mm}(n=79) \\
\text { No. }(\%)\end{array}$ & $\begin{aligned} & \mathrm{MMO} \geq 35 \mathrm{~mm}(n=44) \\
& \text { No. }(\%)\end{aligned}$ & \\
\hline Trismus & $69(87.3)$ & $24(54.5)$ & $3(1.7)$ \\
\hline Burning sensation & $60(75.9)$ & $30(68.2)$ & $23(13.3)$ \\
\hline Xerostomia & $57(72.2)$ & $24(54.5)$ & $24(13.9)$ \\
\hline Maltaste & $18(22.8)$ & $9(20.5)$ & $0(0.0)$ \\
\hline Sore throat & $24(30.4)$ & $11(25.0)$ & $3(1.7)$ \\
\hline Numbness & $18(22.8)$ & $9(20.5)$ & $2(1.2)$ \\
\hline Ulcer & $37(46.8)$ & $21(47.7)$ & $21(12.2)$ \\
\hline Dysphagia & $18(22.8)$ & $3(6.8)$ & $0(0.0)$ \\
\hline
\end{tabular}

$\mathrm{MMO}=$ maximal mouth opening.

TABLE 3

Scoring System for Early Detection of Oral Submucous Fibrosis Derived from Logistic Regression Model Using 6 Symptoms

\begin{tabular}{lr}
\hline Terms & Score \\
\hline Background & 0.17 \\
Clinical symptoms & \\
Trismus & 19.89 \\
Burning sensation & 4.84 \\
Xerostomia & 2.91 \\
Sore throat & 2.72 \\
Numbness & 2.08 \\
Ulcer & 2.57
\end{tabular}

Composite score $=0.17 \prod\left(\right.$ Score $\left._{\mathrm{i}}\right), \mathrm{i}=1$ to $\mathrm{K}$ positive symptoms.

vanced OSF patients (MMO $<35 \mathrm{~mm}$ ). As shown in Table 2, a substantial difference in symptom profiles between early-stage OSF and advanced OSF was noted. To increase the sensitivity for detecting early-stage OSF cases, we excluded 79 severe OSF cases and utilized 44 early-stage OSF cases to develop the scoring system for early detection of OSF.

Excepting the precancerous characteristic, limitation of mouth opening is the most important clinical problem of OSF. Trismus impairs the ability to eat, speak, and even to receive dental treatment. About one-half of our earlystage OSF patients complained of trismus. It seemed that the progression of mouth opening limitation was not so
TABLE 4

Sensitivity and Specificity of Scoring System for Early Detection of Oral Submucous Fibrosis at Various Cutoff Points of Composite Score

\begin{tabular}{|c|c|c|}
\hline $\begin{array}{l}\text { Cutoff } \\
\text { Point of } \\
\text { Composite } \\
\text { Score }\end{array}$ & $\begin{array}{c}\text { Sensitivity } \\
(\%)\end{array}$ & $\begin{array}{c}\text { Specificity } \\
(\%)\end{array}$ \\
\hline 0.2 & 98.5 & 9.1 \\
\hline 0.5 & 86.3 & 75.5 \\
\hline 0.6 & 85.6 & 78.0 \\
\hline 1.0 & 82.0 & 85.8 \\
\hline 1.8 & 77.3 & 92.9 \\
\hline 2.0 & 75.2 & 93.1 \\
\hline 5.0 & 58.2 & 97.7 \\
\hline
\end{tabular}

$\longrightarrow$

insidious among these early-stage OSF patients. In our scoring system for early detection of OSF, trismus had the greatest contribution to the composite score.

The similarity of ROC curves and AUCs between the model including eight symptoms (Figure 1) and the model including six symptoms (Figure 2) suggests little information was lost by dropping maltaste and dysphagia.

While utilizing 123 OSF subjects in the development of the scoring system for identifying OSF cases in general (without regard to $\mathrm{MMO}$ ), the AUC of the ROC curve for the model including six symptoms was 0.942 . The ROC curve for the model including eight 
FIGURE 2

Receiver Operating Characteristic (ROC) Curve for Final Model (Area Under ROC Curve (AUC) $=0.895$ )

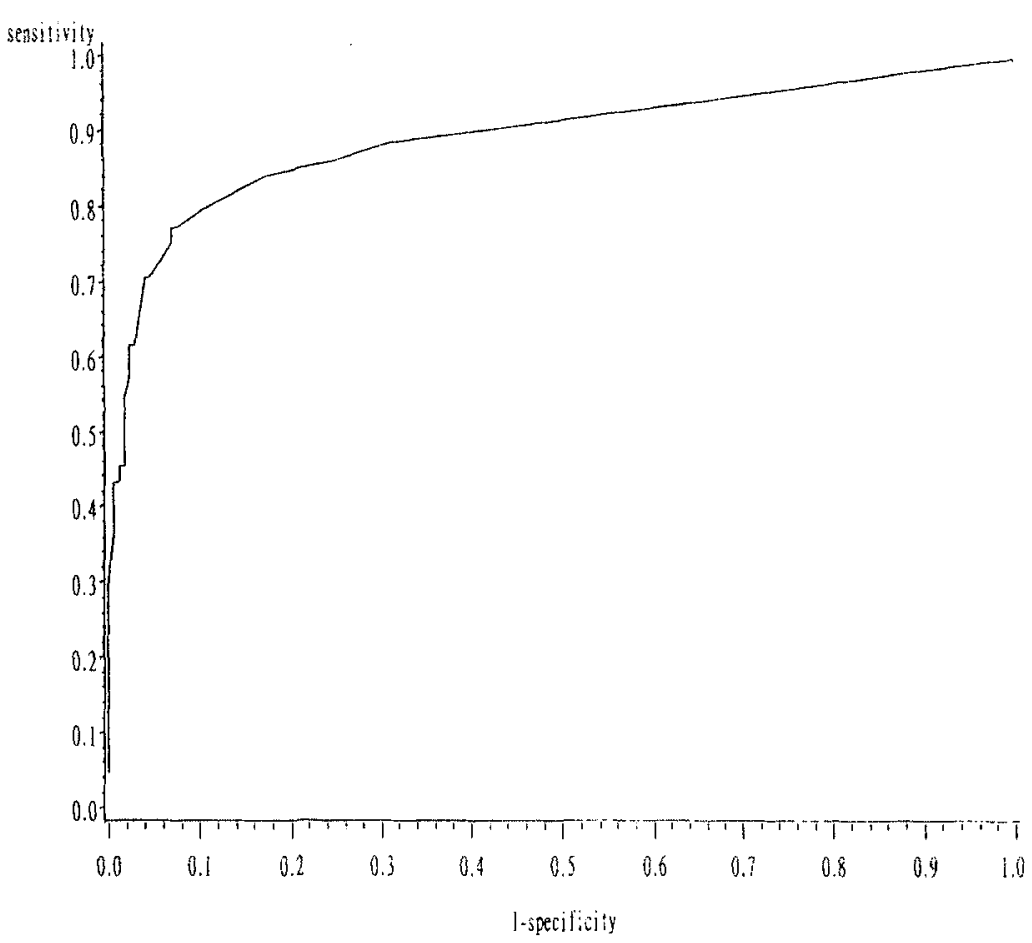

symptoms was also similar to that of the model with six symptoms showing an AUC of 0.946. The general scoring system including six symptoms was composed of background score $(0.087)$, trismus score $(63.86)$, burning sensation score (3.54), xerostomia score (4.26), sore throat score (3.29), numbness score (1.27), and ulcer score (2.57). When the cutoff point of composite score was set as 1.0 , this general scoring system derived from the logistic regression model using six symptoms had sensitivity and specificity of 83.7 percent and 91.9 percent, respectively, for detecting OSF.

As shown in Table 3, it appears that most of the variation can be explained by just two variables: trismus and burning sensation. However, the ROC curve for the model including only trismus and burning sensation showed an AUC of 0.871 for early detection of OSF. When the cutoff point of composite score was set as 1.0 , the sensitivity and specificity of detecting early-stage OSF were 81.8 percent and 86.7 percent, respectively. The ROC curve for the model including only trismus and burning sensation showed an AUC of 0.914 for general (without regard to MMO) detection of tively. ducting the clinical examination dictive value and fewer false positives. On the other hand, we may set a lower cutoff point for screening a high-risk group, such as patients in a BQC special clinic, to reach a higher negative predictive value and fewer false negatives.

The questionnaire we developed here is an easy, self-administered, and noninvasive tool for the early detection of OSF. It could be used in clinics as well as in communities. In conjunction with a two-step design, it could also be used to estimate the prevalence of OSF in the general population. This questionnaire and the scoring system may be used to detect OSF in order to intervene in its progress toward malignant transformation and severe mouth opening limitation. Moreover, symptoms and signs observed in the present study are similar to those reported in the previous studies. This questionnaire is considered to be applicable to other populations because the terms of oral symptoms included in the questionnaire can be easily translated into other languages.

OSF. When the cutoff point of composite score was set as 1.0, the sensitivity and specificity of detecting OSF were 75.6 percent and 98.3 percent, respec-

For calculating sensitivity and specificity here, however, we have applied the scoring system to the same data set from which it has been derived. This does not quite provide sensitivity and specificity, but provides the best possible power of discrimination. To estimate true sensitivity and specificity, the scoring system should to be applied to another series of OSF cases and unaffected BQCs in a blinded fashion. That is, the questionnaire should be completed by subjects before clinical examination and the clinician should not be aware of the results of the questionnaire while con-

As shown in Table 4, the higher the cutoff point of composite score, the lower the sensitivity and the higher the specificity. We may choose an appropriate cutoff point according to the purpose and situation of using the scoring system. For example, we may set a higher cutoff point for screening of a community with low OSF prevalence to obtain a higher positive pre-

\section{References}

1. Pindborg JJ, Murti PR, Bhonsle RB, Gupta PC, Daftary DK, Mehta FS. Oral submucous fibrosis as a precancerous condition. Scand J Dent Res 1984;92:2249.

2. Canniff JP, Harvey W, Harris M. Oral submucous fibrosis: its pathogenesis and management. Br Dent J 1986;160:429-34.

3. Pindborg JJ, Mehta FS, Gupta PC, Daftary DK. Prevalence of oral submucous fibrosis among 50,915 Indian villagers. $\mathrm{Br}$ J Cancer 1968;22:646-54.

4. Cox SC, Walker DM. Oral submucous fibrosis. A review. Aust Dent J 1996;41: 294-9.

5. van Wyk CW, Grobler-Rabie AF, Martell RW, Hammond MG. HLA-antigens in oral submucous fibrosis. J Oral Pathol Med 1994;23:23-7.

6. Cox SC, Walker DM. Establishing a normal range for mouth opening: its use in screening for oral submucous fibrosis. $\mathrm{Br}$ J Oral Maxillofac Surg 1997;35:40-2.

7. Maher $R$, Sankaranarayanan $R$, Johnson NW, Warnakulasuriya KA. Evaluation of inter-incisor distance as an objective criterion of the severity of oral submucous fibrosis in Karachi, Pakistan [letter]. Oral Oncol Eur J Cancer 1996;32B:362-4.

8. Irwig L. Modeling result-specific likelihood ratios. J Clin Epidemiol 1992;45: 1335-6.

9. Hanley JA, Mcneil BJ. The meaning and use of the area under a receiver operating characteristic (ROC) curve. Radiology 1982;143:29-36. 